\title{
Developing an index of restorative dental treatment need
}

\author{
H. C. Falcon, ' P. Richardson, ${ }^{2}$ M. J. Shaw, ${ }^{3}$ J. S. Bulman, ${ }^{4}$ and B. G. N. Smith, ${ }^{5}$
}

\begin{abstract}
The process undertaken to establish an initial pilot index for restorative dental treatment is described. Following consultation with a wide range of clinicians and others, an outline framework for the index was developed and comprised three main components: I. Patient identified need for treatment: the data from the patient perceived need questionnaire were inconclusive; 2 . Complexity of treatment (assessed by clinicians): this was found to be a practical tool capable of being used by a range of dentists. A booklet has been produced which describes the process of using the scoring system; 3. Priority for treatment (assessed by clinicians): three levels of priority were identified; the highest priority was assigned to patients with inherited or developmental defects that justify complex care (eg clefts of the lip and palate). The initial development of the index has had some success in a difficult area. The treatment complexity component is the most developed and may allow both referrers and commissioners of specialist restorative dentistry to determine appropriate use of skilled clinicians' expertise.
\end{abstract}

\begin{abstract}
T the specialty of orthodontics an index 1 of treatment need has been used for some years to categorise patients according to the severity of their malocclusion. ${ }^{1}$ The index allows informed choices to be made by service providers and purchasers regarding types of patients and treatment categories appropriate for inclusion in service contracts.

In the specialty of restorative dentistry no similar index of treatment need exists, although an index which measures oral health status with particular reference to the
\end{abstract}

\footnotetext{
$1^{*}$ Research Fellow, Department of Transcultural Oral Health, Eastman Dental Institute ${ }^{2}$ Director of Research, Defence Dental Agency, RAF Halton, Aylesbury ${ }^{3}$ Consultant in Restorative Dentistry, Birmingham Dental School, St Chad's Queensway, Birmingham B4 6NN ${ }^{4}$ Emeritus Reader, Dental Public Health, Eastman Dental Institute, University College Hospital, 256 Grays Inn Road, London WC1X $8 L D{ }^{5}$ Professor, Conservation Department, Guys Hospital Dental School, London Bridge, London SE1 9RT

${ }^{*}$ Correspondence to: H C Falcon, Aylesbury Vale Community NHS Trust, Manor House, Bierton Road, Aylesbury HP20 1EG

email: Helen.Falcon@btinternet.com

REFEREED PAPER

Received 13.01.00; Accepted 11.10.00

(C) British Dental Journal 2001; 190: 479-486
}

adequacy of dental restorations has been described. ${ }^{3}$ The Clinical Audit Committee of the Faculty of Dental Surgery, Royal College of Surgeons of England identified the requirement for such an index and obtained funds from the Department of Health to support a development project. The project was administered by the Audit Working Group for Restorative Dentistry and a research team was established following approval from the Department of Health. The aim of the project was to establish an initial pilot index that would allow need to be identified and ranked by complexity. The index would have to be simple to use, based on evidence, reproducible and produce patient-based data that would be

\section{In brief}

- Levels of complexity of treatment were determined for endodontics, periodontics, fixed and removable prosthodontics.

- Modifying factors relate to patient management.

- Priority for specialised treatment was related to the complexity of providing care. amenable to statistical analysis. It was intended that the index, once developed might be used in a similar way to the orthodontic index, both to inform purchasers during contract negotiations and to audit referral and clinical activity.

This paper describes the process undertaken to establish an index of treatment need for restorative dentistry. It outlines the structure of the complexity component of the index, provides details of the codes agreed and summarises the progress in developing contributory components .

\section{Background}

Dental indices that measure need for treatment rather than dental health status are not widely used in the UK. The exceptions are the Index of Orthodontic Treatment Need $^{1}$ and the Community Periodontal Index of Treatment Need. ${ }^{2}$ Each has been used as both a population based measurement and, with modification, as an individual treatment planning tool. They enable treatment complexity as well as past disease activity to be quantified. IOTN incorporates a patient-assessed component and records clinician assessed treatment need, but its scoring system does not give an indication of treatment complexity. Neither index addresses the aetiology of the condition but merely seeks to measure the need for intervention. Each index enables audit of patient selection and permits some limited analysis of treatment outcome.

It was decided to design a restorative dental index incorporating some of the features of these two well-used and evaluated examples.

\section{Establishing and testing the}

framework of the index

Two researchers, (HF, PR) contacted and then visited eight restorative dental departments in teaching hospitals and one district general hospital within the UK. In addition, they met with senior representatives of the British Dental Association, Community Dental Service, Faculty of General Dental Practitioners, Dental Practice Board, Dental Protection Societies and Consultants in Dental Public Health. The aim was to establish an understanding of the key issues relating to referrals for restorative dental care 

four categories in each of 17 cases

All ex 14 examiners (*except RCT which is ex 13)

\begin{tabular}{lccccccc} 
& Unanimous & I dissent & 2 dissent & 3 dissent & 4 dissent & $>4$ dissent & Total \\
Perio & 2 & 1 & 3 & 2 & 2 & 7 & 17 \\
RCT & 12 & 1 & 0 & 0 & 1 & 2 & 16 \\
Pros-remove & 10 & 1 & 0 & 2 & 1 & 3 & 17 \\
Pros-fixed & 7 & 2 & 1 & 2 & 3 & 14 & 67 \\
Total & 31 & 5 & 4 & 6 & 7 & & 17 \\
\hline
\end{tabular}

and to ensure any resulting index took these views into account.

An outline framework for the index was developed and comprised three main components:

1 Patient identified need for treatment

2 Complexity of treatment (assessed by clinician)

3 Priority for treatment (assessed by clinician)

An outline of the proposed index, together with several clinical case reports to illustrate the index structure, was presented to the consultants in restorative dentistry group at an annual meeting. The aim was to seek co-operation and promote consensus prior to establishing draft codes and initial boundaries to rank priority and complexity. It was decided to achieve this initially with a series of questionnaires circulated to clinicians within the specialty. Nominees from the relevant specialist societies (British Society for Restorative Dentistry, British Society for the Study of Prosthetic Dentistry, British Endodontic Society, British Society for Periodontology) contributed to the questionnaire design and content. The questionnaires identified a range of treatment procedures undertaken in restorative dentistry and asked respondents to score each treatment process separately for priority and complexity.

A postal questionnaire was distributed to 141 members of the consultants in restorative dentistry group. The questionnaire was divided into sections relating to the socalled 'mono specialities' within restorative dentistry (periodontics, endodontics, fixed and removable prosthodontics). Some consultants, with a known mono-specialty interest, were sent only sections of the questionnaire that were relevant to their own clinical activity. A $66 \%$ return was achieved from those circulated.
Fig. I Assessment procedure

Each component of the assessment should be considered separately and may be the only relevant component for that patient. A complexity code should be assigned, if applicable, before progressing to the next component. Each complexity component has a series of three codes (low $=\mathrm{I}$, moderate $=2$, high = 3 ) ( Figs 3-6 ) to which a modifying factor may apply (Fig. 7). Modifying factors are similar for each component of the index, although there are some variations; they should be applied to each component of the index.

Periodontal assessment (if relevant) + modifying factor (if applicable)

Periodontal treatment complexity code<smiles>[CH]1C=C1</smiles>

RCT assessment (if relevant) + modifying factor (if applicable)

\section{RCT complexity code}

Fixed prosthodontics assessment (if relevant) + modifying factor (if applicable)<smiles>C1=C[C@H]2CC[C@H]1C2</smiles>

Fixed prosthodontics complexity code

Removable prosthodontics assessment (if relevant) + modifying factory (if applicable)

Removable prosthodontics complexity code

highest code $=$ restorative dentistry complexity code 


$\begin{array}{lcccccr}\text { Agreement } & \text { N/A* } & \text { Grade I } & \text { Grade 2 } & \text { Grade I-2 } & \text { Grade 3 } & \text { Total } \\ 100 \% & 20 & 1 & 0 & 0 & 0 & 21 \\ \text { Majority } & 12 & 6 & 5 & 2 & 7 & 32 \\ 50 \% & 0 & 2 & 1 & 0 & 1 & 4 \\ \text { Minority } & 0 & 5 & 5 & 0 & 0 & 10 \\ \text { Total } & 32 & 14 & 11 & 2 & 8 & 67\end{array}$

*Refers to categories where a pathological condition is deemed to be not present

Table 3

Degree of diagnostic agreement between 14 practitioners and anticipated outcome

in terms of categories and cases

\begin{tabular}{|c|c|c|c|c|c|}
\hline Agreement & $\begin{array}{c}\text { Periodontal } \\
\text { treatment }\end{array}$ & $\begin{array}{l}\text { Root canal } \\
\text { treatment }\end{array}$ & $\begin{array}{c}\text { Prosthetics- } \\
\text { removable }\end{array}$ & $\begin{array}{l}\text { Prosthetics- } \\
\text { fixed }\end{array}$ & Total \\
\hline $100 \%$ & I & 9 & 8 & 3 & 21 \\
\hline Majority & II & 5 & 5 & II & 32 \\
\hline $50 \%$ & 2 & 2 & 0 & 0 & 4 \\
\hline Minority & 3 & 0 & 4 & 3 & 10 \\
\hline Total & 17 & 16 & 17 & 17 & 67 \\
\hline
\end{tabular}

Data were analysed from the returned questionnaires and found to be sufficiently consistent to develop an initial series of codes and boundaries (low, moderate, high) for the complexity component of the index for dentate patients. The edentate were seen, from the questionnaires, to present a different complexity ranking task to the dentate. The data from the priority sections of the questionnaires were inconclusive.

\section{Complexity component}

The returned questionnaires allowed a draft complexity index to be compiled, listing a series of restorative procedures as either:

Complexity code 1 Able to be performed by any dental graduate

Complexity code 2 Able to be performed by any experienced dentist

Complexity code 3 Able to be performed by any dentist with skills developed following specialist training

This first draft of the index was refined by a group of consultants and specialist trainees in a series of structured discussions. The subsequent draft was taken to a group of ten dentists from across the UK, consisting of vocational trainers, specialist practitioners and hospital consultants. The clinicians were involved in a facilitated discussion to further refine the components of the index. A final version of the complexity component was agreed and, in order to determine the feasibility of using the index, it was then applied by the same group of dentists to clinical data. These comprised clinical information, study models and radiographs. The index was found to be an appropriate and practical tool and feedback was obtained about layout, wording and presentation.

\section{Reproducibility exercise}

A training and reproducibility exercise was organised at the Dental Practice Board in Eastbourne. A group of 14 clinicians from general dental practice, community dental practice and the dental practice board took part in a training and reproducibility 


\section{\begin{tabular}{|l|l|}
\hline Fig. 2 & Terminology used in the complexity assessment \\
\hline
\end{tabular}}

The complexity codes use certain terminology in defining the scoring criteria:

\section{BPE - basic periodontal examination}

The basic periodontal examination requires that the periodontal tissue should be examined with a standardised periodontal probe using light pressure, Examine the tissues for bleeding, plaque retentive factors and pocket depth:

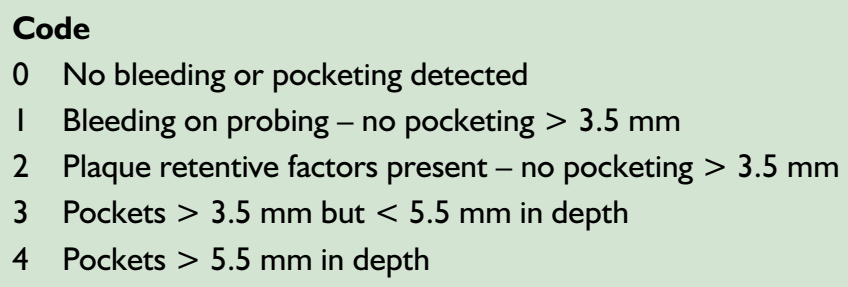

In the BPE all teeth are examined and the worst (highest) score in each sextant is recorded.

\section{Anterior guidance}

Anterior Guidance is the anterior determinant of mandibular movement. It can be provided by any tooth or group of teeth that guide forward or lateral movement of the mandible.

\section{Conformative treatment}

Conformative dental treatment is treatment that conforms to the existing intercuspal position.

\section{Reorganised dental treatment}

Reorganised treatment is dental treatment that changes the existing intercuspal position.

\section{Terminal unit}

The terminal unit is the most distal tooth in any quadrant.

exercise to test the inter examiner variability of the complexity component of the index. Following an introductory training session, involving an explanation of the codes, presentation of clinical examples and a hands-on training exercise, each participant applied the index to 17 sets of clinical data. They were asked to assign a complexity score to each data set. The resultant data were analysed, comparing the codes assigned by each participant with the codes previously agreed by the research team. One set of clinical data was duplicated and unknowingly scored twice to measure intra examiner variation.

\section{Results}

The results showed no marked contrasts or trends between the three practitioner groups and so they were combined. The mean time for the assessment of each data set was 6.1 minutes. There was a mean conformity coefficient (a measure of the level of agreement of scores between participants) for the group of $80 \%$. (Range $64 \%-96 \%)$. With 17 cases and four categories per case (periodontics, root canal treatment (RCT), fixed prosthodontics and removable prosthodontics), there were theoretically 68 possibilities for unanimous agreement. However, one participant omitted a RCT score for one patient, bringing the total possible number of recorded scores to 67 . There were 31 out of 67 records of unanimous agreement among the 14 participating practitioners. A more detailed breakdown is shown in Table 1, where results are itemised accord- ing to category. This shows that there were two or fewer dissenters from a unanimous diagnosis in 40 out of 67 records $(60 \%)$, and four or fewer dissenters in 53 out of 67 records $(79 \%)$.

When the decisions reached by the participants were compared with those anticipated by the project team, in terms of grading (ie not applicable and grades 1-3), there was a unanimous or a majority agreement in 32 out of 32 instances of a 'not applicable' diagnosis, 7 out of 14 instances of a 'Grade 1' diagnosis, 2 out of 2 of a 'Grade 1-2', 5 out of 11 of a 'Grade 2', and 7 out of 8 of a 'Grade 3', giving a total of 53 out of 67 majority agreements. A more detailed breakdown is given in Table 2 .

In terms of categories and cases there was majority agreement (over 50\%) in 12 out of 17 periodontal categories, 14 out of 16 RCT categories, 13 out of 17 removable prosthodontics categories and 14 out of 17 fixed prosthodontics categories. Table 3 contains a more detailed breakdown.

\section{The assessment process}

A booklet was designed describing the process of applying the complexity assessment (Figs. 1, 2). The assessment process involves examining the patient and applying a complexity code (range 1-3) for each component of the examination that relates to restorative dentistry. The complexity codes are divided into four main components (Figs. 3-6), involving a periodontal treatment assessment, a root canal treatment assessment, and separate assessments in fixed and removable prosthodontics. Each component of the assessment should be considered separately and may be the only relevant component for the complexity assessment for that patient. Each complexity component has a series of clinical descriptors that are ranked as low (code 1), moderate (code 2) or high complexity (code 3 ); to these a modifying factor may apply. The modifying factors (Fig. 7) are similar for each component of the index although there are small variations. They are predominantly related to patient management issues. Modifying factors only increase complexity by one code increment; they are not 
cumulative. The highest complexity is code 3.

\section{How to use the complexity assessment tool}

\section{Step 1}

Following a clinical examination and assessment of the patient's oral status, appropriate codes are selected which most closely describes the patient's condition or treatment requirement.

For example, a patient with a BPE score of 3 in one sextant, a non vital molar tooth with one root canal curvature greater than 40 degrees to the root long axis and requiring $a$ mucosal born partial prosthesis would be scored

\section{Periodontal complexity 1 \\ Root canal treatment complexity 3 \\ Prosthodontic complexity 1}

\section{Step 2}

The highest code achieved in any of the individual components is the overall restorative dentistry complexity score

In the example given, there would be an overall restorative dentistry complexity code of 3 .

\section{Step 3}

The complexity codes are then recorded on a data collection sheet, together with an indication of whether modifying factors apply. Application of a modifying factor increases the score by one increment, unless the maximum score has already been achieved. This is the final restorative dentistry complexity score.

In the example given the highest complexity score (3) has already been achieved and an additional modifying factor relating to the management of the patient would not change the level of complexity assessment.

\section{Priority component}

The questionnaires referred to in the first phase of the project failed to give any clear indication of priority for specialist treatment, either in relation to patient category, or dental procedure.

A workshop was held involving a group of 10 clinicians including general dental

\section{Fig. 3} Component I: periodontal treatment assessment

Based upon the basic periodontal examination (BPE) criteria

- BPE score I - 3 in any sextant

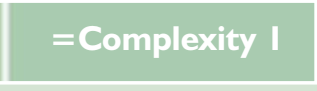

- BPE score of 4 in any sextant

- Surgery involving the periodontal tissues

- Surgical procedures associated with osseointegrated implants

- Surgical procedures involving periodontal issue augmentation and/or bone removal (eg crown lengthening surgery).

- BPE score of 4 in any sextant and including one or more of the following factors:

Patients under the age of 35

Smoking $10+$ cigarettes daily

A concurrent medical factor that is directly affecting the periodontal tissues

Root morphology that adversely affects prognosis

Rapid periodontal breakdown

$>2 \mathrm{~mm}$ attachment loss in any one year practitioners, community dentists, consultants in restorative dentistry and in dental public health. Prior to the group work all participants agreed some fundamental principles:

- Any patient should have access to consultant services for advice or treatment planning guidance, following appropriate referral from a primary care practitioner.

- Any patient should have equal access to specialist care irrespective of socio-economic group, gender, age or racial origin

- Only those patients for whom treatment was of sufficient complexity to justify care from a trained specialist (assessed as part of the complexity coding) should receive care.

Participants were divided into two groups and asked to agree whether there were any categories of patients who should receive priority for restorative dentistry specialist treatment. Subsequent to this initial task the two groups were asked to debate and if possible agree whether there were any aspects of treatment or particular groups of teeth that should be considered a priority for specialist care. The two groups then shared their ideas and attempted to reach a consensus about the priorities raised.

\section{Results - categories of patients}

The two working groups considered that many of the patient groups that could be considered a priority category would already be scored as such via the modifying factors applied in the complexity assessment process.

The following patient categories were ranked as a priority for specialist restorative dental treatment:

\section{Priority One}

- Patients with inherited or developmental 


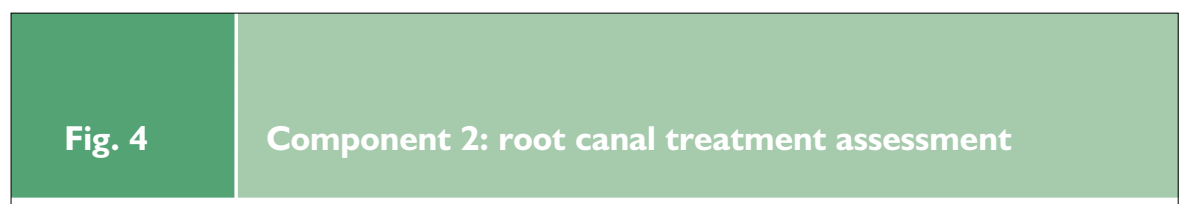

Root canal treatment assessment (permanent teeth)

Conventional root canal treatment or retreatment is the clinical procedure of choice. Surgical treatment should only be considered when conventional treatment is inappropriate.

- Single/multiple root canals with curvature $<15^{\circ}$ to root axis that are considered negotiable from radiographic or clinical evidence through their entire length. No root canal obstruction or damaged access

- Surgical treatment

- Single root canals

- Radiolucency $<6$ mm diameter

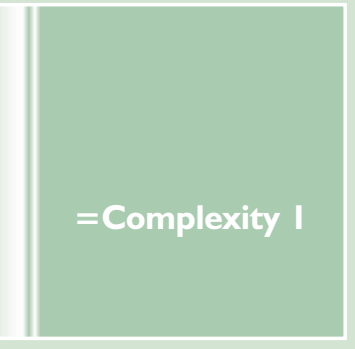

- Single/multiple root canals with curvature $>15^{\circ}$ but $<40^{\circ}$ to root axis that are considered negotiable from radiographic or clinical evidence through their entire length.

- Surgical treatment

- Single root canals

- No evidence of radiolucency

- Hemisection of mandibular molars

- Teeth with incomplete root development

- Single/multiple root canals with curvature $>40^{\circ}$

- Single/multiple root canals that are not considered negotiable from radiographic or clinical evidence through their entire length

- Surgical treatment

- Multi rooted teeth

- Single root canals

- Radiolucency > 6 mm diameter

- Teeth with iatrogenic damage or pathological resorption

- Teeth with difficult root morphology

$\begin{array}{ll}15^{\circ} \text { to the } & 40^{\circ} \text { to the } \\ \text { root axis } & \text { root axis }\end{array}$
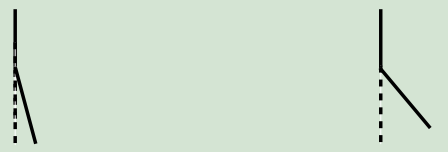

defects that justify complex care (eg clefts of the lip and palate, hypodontia, amelogenesis imperfecta, etc.)

\section{Priority Two}

- Those who have previously received complex care that requires maintenance or replacement (assessed as part of the complexity assessment process).

- When tooth or tissue loss has occurred as the result of trauma.

- People with special needs, including those with learning difficulties, physical disabil- ities, social impairment, mental illness or who are medically compromised.

- When multi-specialty working is indicated.

- Those receiving oncology treatment that has implications for oral and dental care, (to include those people requiring post surgical reconstruction with a fixed or removable prosthesis).

- Those for whom a lack of specialist treatment may affect their livelihood (eg musicians).

- Where specific facilities were required to enable treatment (eg in patient or day stay care requiring general anaesthetic facilities with specialist anaesthetist support).

\section{Reduced priority}

The working groups agreed that there were certain factors that would reduce priority for specialist care:

- Lack of motivation or commitment to comply with preventive regimens, although it was acknowledged that certain patient groups may have difficulties complying and special consideration should be given to them.

- Smokers in certain categories of care, eg periodontal therapy, implant treatment.

\section{Results - types of treatment}

Both the working groups agreed the following objectives when establishing priorities in restorative dentistry:

- To maintain 20 functioning, aesthetic and predictably healthy teeth.

- To maintain sufficient teeth in each dental sextant to provide a foundation for a fixed or removable prosthesis, where possible avoiding a free end saddle appliance.

\section{Patient assessed need component}

The Index of Orthodontic Treatment Need incorporates a patient assessment of need via an aesthetic component. This was designed following the construction of an illustrated 10-point scale using dental photographs of 12-year-old children and rated by six non-dental judges. In restorative dentistry there are other factors that affect patient assessed need including oral pain or discomfort, difficulties when eating or social embarrassment. An aesthetic assessment was considered and rejected, since it would only have reflected part of the patients' assessed component of need.

Other measures of patient assessed need have been developed, and largely focus on the impact of oral disorders on an individual, rather than an attempt to measure need for a particular intervention, since it is argued that most patients are not able to comment on the latter. ${ }^{5,6}$ One measure which has been adopted for measuring the 


\begin{tabular}{l} 
Fig. 5 \\
This basic assessment assumes that the proposed restorative dental treatment \\
will conform to the existing occlusion. The principles apply to conventional and \\
adhesive units. \\
Fixed Prosthodontic restorations include: \\
- Intra coronal restorations \\
- Veneer restorations \\
- Extra coronal restorations including pontic units \\
- Restorations not involved in anterior guidance, \\
where there are adequate sound or restored teeth \\
to predictably maintain the existing occlusion \\
\hline - Restorations that contribute to anterior guidance || Complexity | \\
where there are insufficient sound or restored \\
teeth to predictably maintain the current \\
guidance \\
- Extra coronal restoration of any one posterior \\
sextant (all teeth), not involved in anterior \\
guidance where a terminal unit is involved \\
- Extra coronal restoration of the complete anterior || \\
- Euidance including pontic units. \\
integrated implants \\
\hline
\end{tabular}

member of clinical audit staff. Analysis of both the initial questionnaires and when they were repeated, indicated inconsistent results that would require a further study with a larger patient sample to establish whether this form of self reported profile analysis could yield an appropriate measurement tool.

\section{Discussion}

This initial development of an index of treatment need for restorative dentistry has had some success in a difficult area. The development of the complexity component of this index will help to initiate a debate concerning treatment complexity and as the only complexity index for restorative dentistry currently, may find sufficient support to be used both to inform the referrer, provider and purchaser about specialty care. There is potential for it to be used as a quality monitoring indicator and it may have a role in clinical governance for spe- impact of oral disorders with different groups of patients is the Oral Health Impact Profile (OHIP). ${ }^{7}$

It was decided to pilot a simplified version of the OHIP as a basis for the development of the patient assessed need component, since it contained a wide range of indicators relevant to restorative dentistry and had been widely tested.

A questionnaire based on the Oral Health Impact Profile was developed and used to conduct structured interviews at three dental teaching hospitals and one district general hospital across England.

A total sample of 171 patients attending for their first out patient appointment were asked a series of questions about the problems they experienced with function (eg eating, painful mouth), specific toothrelated issues (eg food impaction, sharp or broken teeth) and psycho-social issues (eg embarrassment, sleep disturbance). Before the initial consultation, the participants were asked to quantify their problem using a visual analogue (Likert) scale. All patients attending the participating specialist clinics on the selected days were invited to take part. The questionnaires were administered by one of the project team (HF) or a trained
Fig. 6

\section{Component 4: removable prosthodontics treatment assessment}

Basic assessment assumes that the proposed treatment will conform to the existing occlusion

- Prostheses with bounded saddles replacing posterior teeth.

- All mucosal born prostheses.

- Prostheses replacing anterior teeth where there are adequate sound or restored teeth to provide anterior guidance.

- Free end saddle prostheses which are dependent upon differential support.

- Prostheses with problems involving the path of insertion and/or available undercuts where some tooth modification is involved.

- Prostheses which contribute to anterior guidance.

- Prostheses where abutment teeth require extra coronal restoration to improve stability and retention.

- The use of sectional prostheses.

- Prostheses involving osseo integrated implant support.

- Presence of oro-facial defects requiring obturation/restoration. 
Modifying factors that are relevant to each complexity assessment

A modifying factor can only increase complexity by one increment. Multiple factors are not cumulative.

- Co-ordinated medical (eg renal: cardiac) and/or dental

(eg oral surgery : orthodontic) multi-disciplinary care

- Medical history that significantly affects clinical management (*See below)

- Special needs for the acceptance or provision of dental treatment

- Mandibular dysfunction

- Atypical facial pain

- Undiagnosed facial pain

- Presence of a retching tendency

- Limited operating access

\section{Modifying factors that are relevant to specific components of the complexity} assessment:

Relevant to Component I: periodontal treatment assessment

- Concurrent mucogingival disease (eg lichen planus): xerostomia

Relevant to Component 2: root canal treatment

- Root canal treatment

- Surgery in the proximity of important anatomical structures eg mental foramen

- Surgery when periodontal attachment loss exeeds $3.5 \mathrm{mms}$.

Relevant to Component 3 and 4: prosthodontic assessments

- Concurrent mucogingival disease (eg lichen planus): xerostomia

- Skeletal base discrepancy that affects the occlusion

- Evidence of significant parafunction

- Reorganisation of the occlusion required

- Radiographic evidence of $50 \%$ reduction in bone support

Medical history that significantly affects clinical management

- Patients requiring IM or IV medication as a component of clinical management.

- Patients with a history of head/neck radiotherapy.

- Patients who are significantly immuno compromised or immuno suppressed.

- Patients with a significant bleeding dyscrasia/disorder.

- Patients with a potential drug interaction.

cialist restorative dental care. The process involved in designing the complexity component and the reproducibility exercise would suggest that it is a practical, easy to use complexity assessment tool. Its validity has been demonstrated in ideal conditions with a small representative group of clinicians, but will need to be extensively tested with a much larger patient group, using live clinical examinations and a greater number of clinicians from diverse backgrounds. It is likely that it could be a useful tool for assessing individual adults requiring restorative treatment, but would need to be tested on a whole population sample before recommendations could be made about its applicability in general epidemiological surveys. The individual criteria used are relatively simple and easily understandable. Where possible they have been based on accepted and validated measures, for example CPITN; but the grades of complexity are based on collective clinical opinion, taken from both the questionnaire and subsequent modification by the working groups. Further studies are needed on clinical outcomes for restorative procedures carried out by clinicians with differing experience and training, and these should inform the complexity bandings, which currently are subjective.

Whilst restorative dental treatment is largely mechanistic, patient management also involves both bio-medical and psychological assessment. An attempt has been made to address this through the modifying factors.

When taken as a whole, the final complexity score can only represent an indicative, broadly based measure of complexity, but the individual elements of the composite index offer a more precise measure, although with the same caveats as before.

The priority working group proposed a number of groups of patients for specialist care and described those who were either more vulnerable or who more frequently appeared to need help from specialist advice, treatment or facilities. They largely reflected current priorities in NHS specialist practice.

The use of the Oral Health Impact Profile has promise in developing a patient initiated measure of perceived need. Patients perceive their needs to be related to comfort, function and appearance. Dentists use similar criteria but place more emphasis on the presence of disease to prioritise need. The highest values in the scoring of treatment need will therefore always be attributable to conditions causing pain or difficulties with eating or speaking, in addition to an unacceptable dental appearance. Frequently such problems can be resolved within primary dental care without requiring specialist help. The need for specialist help becomes apparent when the care that is required is outside the experience, abilities or facilities available to the referring primary care dentist. The need for specialist care is therefore more often related to issues of complexity of care, giving an element of priority to those patients who have more complex management requirements.

A booklet outlining the complexity component of the index is available from the authors.

1 Brook P H, Shaw W C. The development of an index of orthodontic treatment priority. Eur J Orthod 1989; 11: 309-320.

2 Ainamo J, Barmes D, Beagrie G, Cuttress T, Martin J, Sardo-Infirri J. Development of the World Health Organization (WHO) community periodontal index of treatment need (CPITN). Int Dent J 1982; 32: 281-291.

3 Burke F J T, Wilson N H F. Measuring Oral health: an historical view and details of a contemporary oral health index (OHX). Int Dent J 1995; 45: 358-370.

4 Cushing A M, Sheiham A, Maizels J. Developing sociodental indicators - the social impact of dental disease. Community Dent Health 1986; 3: 3-17.

5 Atchison K A, Dolan T A. Development of the geriatric oral health assessment index. J Dent Educ 1990; 54: 680-687.

6 Locker D. Measuring oral health: a conceptual framework. Community Dent Health 1988; 5: 3-18.

7 Slade G D, Spencer J. Development and evaluation of the oral health impact profile. Community Dent Health 1994; 11: 3-11. 